\title{
Obtenção de fosfatos de cálcio pelo método biomimético sobre a superfície da liga Ti-6Al-4V modificada pelo laser Nd:YAG
}

\author{
Calcium phosphates obtained \\ by the biometric method onto the Ti-6Al-4V \\ alloy surface modified by a Nd:YAG laser
}

Edson Almeida Filho ${ }^{1}$, Alexandre Félix Fraga ${ }^{1}$, Rafael Admar Bini ${ }^{2}$, Antonio Carlos Guastaldi ${ }^{\text {I }}$

\footnotetext{
${ }^{1}$ Instituto de Química, Grupo de Biomateriais Universidade Estadual Paulista - UNESP, rua Francisco Degni, s/n, CEP 14800-900, Araraquara, São Paulo, Brasil.

e-mail: edsonafilho@yahoo.com.br; affraga@yahoo.com.br; guastald@iq.unesp.br.

${ }^{\text {I } 2}$ Instituto de Química de São Carlos, Grupo de Materiais Coloidais, Universidade de São Paulo. av. Trab. Sancarlense, 400, cp. 780, São Carlos, São Paulo Brasil.

e-mail: r_bini11@yahoo.com.br
}

\section{RESUMO}

Nesse trabalho, estudou-se a modificação da superfície da liga Ti-6Al-4V pela irradiação com feixe de laser Nd:YAG, variando o espaçamento da matriz e mantendo-se constantes os demais parâmetros. Após a irradiação, as amostras foram imersas na solução SBF (Simulated Body Fluid) por 7 dias a $37^{\circ} \mathrm{C}$, para a nucleação e formação das apatitas. Após o recobrimento, essas amostras foram submetidas a um tratamento térmico a $600{ }^{\circ} \mathrm{C}$. Resultados de difração de raiosX indicaram a formação de uma mistura de fases contendo hidroxiapatita e fosfato tricálcico. Análises no infravermelho constataram a presença de estiramentos referentes à hidroxiapatita. As micrografias mostraram que diferentes espaçamentos da matriz proporcionaram a formação de diferentes morfologias. $\mathrm{O}$ espaçamento de $0,01 \mathrm{~cm}$ induziu a formação de microesferas características da fase hidroxiapatita, enquanto, para o espaçamento $0,02 \mathrm{~cm}$, apenas um recobrimento não homogêneo foi obtido. Portanto, a utilização de laser na produção de superfícies ativas para a deposição de biocerâmicas mostrou-se viável, e o substrato após o recobrimento final possui potencial para aplicações na área de implantes dentários.

Palavras-chave: Liga Ti-6Al-4V. laser Nd:YAG. hidroxiapatita. implantes odontológicos.

\section{ABSTRACT}

In this work, surface modification of the Ti-6Al-4V alloy by irradiation with a Nd:YAG laser beam was studied. The matrix spacing was varied and the other parameters were kept constant. After theirradiation, the samples were immersed into SBF solution (Simulated Body Fluid) for 7 days at $37^{\circ} \mathrm{C}$ for nucleation and formation of calcium phosphates. After this period, the samples were subjected to a heat treatment at $600{ }^{\circ} \mathrm{C}$. $\mathrm{X}$-ray diffraction results indicated the formation of a mixture of phases containing hydroxyapatite and tricalcium phosphate. Infrared analysis demonstrated the presence of bands related to hydroxyapatite. SEM micrographs showed that different matrix spacing used leads to the formation of different morphologies.The $0.01 \mathrm{~cm}$ spacing induced the formation of microspheres, characteristic of the hydroxyapatite phase, while for $0.02 \mathrm{~cm}$ spacing, only a non-homogeneous coating was obtained. Therefore, the use of laser in the production of active surfaces for deposition of bioceramics proved to be viable, and the final coating has potential for applications in the field of dental implants.

Keywords: Nd:YAG laser, Hydroxyapatite, Ti-6Al-4V alloy, Dental implants. 


\section{INTRODUÇÃO}

Implantes dentários enquadram-se na definição de biomateriais. Em um sentido amplo, um biomaterial pode ser definido como qualquer substância, excetuando-se as drogas, que pode ser usada, por qualquer período de tempo, como parte de um sistema que trata, aumenta ou substitue quaisquer tecidos, órgãos ou funções do corpo $[1,2]$. O titânio e suas ligas, como, por exemplo, a Ti-6Al-4V, são materiais amplamente usados em cirurgias ortopédicas e odontológicas, devido às propriedades físicas especiais obtidas pelo balanço das fases alotrópicas decorrentes do processamento e da contribuição dos estabilizantes de fases, como baixo módulo de elasticidade, baixo peso e baixa condutibilidade térmica. Sua excelente biocompatibilidade está associada à fina camada superficial de óxido de titânio com espessura de 4 a 6 nanômetros, que se forma espontaneamente quando exposta ao ar, ou artificialmente mediante técnicas de passivação. Este óxido é extremamente aderente e eletroquimicamente inerte, e tais características o tornam um material altamente resistente à corrosão [3].

A ciência da superfície teve um desenvolvimento revolucionário nas últimas décadas, principalmente sobre técnicas de preparação e modificação de superfícies, possibilitando deposição de materiais similares por processos físicos e químicos. Somente após esse avanço, foi possível a evolução dos estudos de implantes a partir de superfícies modificadas, com tensão superficial adequada, que propicia o seu molhamento pelo nutriente, obtida por meio de processos químicos [3, 4]. O longo período da ligação interfacial entre um implante e o osso pode ser melhorado pela criação de uma superfície rugosa, ou de um filme de natureza porosa no implante, o que permite aumentar a área superficial disponível para a justaposição osso-implante e aumentar a osseointegração. A osseointegração é um processo pelo qual ocorre a formação de um tecido ósseo vivo em torno do implante, de forma previsível e duradoura, estabelecendo um contato mecânico que resulta na ancoragem desse implante [4].

Várias técnicas são utilizadas com o objetivo de construir uma superfície totalmente favorável não só à osseointegração, mas também ao depósito de apatitas. Dentre essas técnicas, a modificação por feixe de laser está apresentando resultados satisfatórios quando comparada aos tratamentos utilizados comercialmente (ataque ácido e aspersão térmica), apresentando-se, assim, como uma técnica promissora para modificações de superfícies com esses fins. Recentemente, foi descoberto que o laser Nd:YAG pode obter potências na faixa de kilo-Watts no estado sólido. O elemento ativo $\mathrm{Nd}^{3+}$ gera feixes com comprimento de onda de 1,06 $\mu \mathrm{m}$. Esse comprimento de onda permite o transporte do feixe por meio de fibras óticas. Em princípio, o laser Nd:YAG opera em um modo pulsado, porém em altíssimas frequências, que o aproximam de um modo contínuo. A principal desvantagem desse tipo de laser está em sua baixa eficiência: cerca de $0,2 \%$ a $3 \%$ [14]. Outro tipo de modificação é uso de biocerâmicas de fosfato de cálcio. Essa classe de biomateriais é utilizada em procedimentos para a reconstrução e recomposição tecidual, devido às suas características e propriedades (principalmente a biocompatibilidade e osteocondutibilidade), e ainda por sua semelhança estrutural, química e física com a matriz mineral óssea [5]. As biocerâmicas não induzem qualquer reação imunológica ou tóxica quando utilizadas, bem diferente de alguns materiais que têm origem orgânica. Não apresentam riscos de transmissão de patologias infectocontagiosas nem de degradação protéica, em razão de suas características e por apresentarem alta pureza em decorrência de seu processo de obtenção com rígido e sistemático controle de acordo com os padrões exigíveis [6].

Em meio aos diversos materiais de fosfato de cálcio, existe um que merece destaque maior, por ser o principal constituinte da fase mineral dos tecidos calcificados: é a hidroxiapatita (HA). A HA é uma biocerâmica que tem uma estrutura similar à fase mineral de ossos e dentes [7]. Este material possui propriedades como biocompatibilidade, bioatividade e osseointegração. As propriedades químicas da HA podem ser modificadas através do método de sua preparação. Para implantes ósseos ou dentários, a hidroxiapatita é utilizada por apresentar baixa solubilidade no meio biológico. Quando se deseja que o implante seja reabsorvido pelo corpo, cedendo lugar ao tecido ósseo novo, utiliza-se uma cerâmica mais solúvel, geralmente constituída por uma mistura de hidroxiapatita com outros fosfatos de cálcio [8]. Esses outros fosfatos podem ser transformados em cerâmicas osteocondutoras (cerâmicas com capacidade para fazer com que o crescimento ósseo ocorra sobre a superfície dos poros do material) [9].

Com a descoberta das propriedades da HA, pesquisadores como ABE et al. (1990) [10] introduziram o método biomimético, uma das técnicas mais promissoras para a produção de biomateriais em condições ambientes. Devido a essas condições, é possível recobrir materiais de formas complexas, como materiais porosos, e também materiais sensíveis à temperaturas, como é o caso dos polímeros. Além disso, com essa técnica, pode-se recobrir implantes com diferentes fases de fosfatos de cálcio, as quais possuem características benéficas para formação óssea $[6,10,11]$. SHI et al. resolveram modificar a superfície utilizando a técnica de plasma-spray para o depósito de uma camada de titânio comercialmente puro (Ti-cp) 
sobre o substrato de Ti-6Al-4V. Após o depósito da camada, o substrato recebeu um tratamento em uma solução de hidróxido de sódio. Obteve-se, sobre a superfície, uma camada do hidrogel à base de titanato de sódio [12]. Recentemente, tem sido estudada a modificação da superfície do titânio durante o tratamento químico e, posteriormente, o depósito de HA pela solução SBF. Com o avanço nos estudos da formação da apatita sobre metais, achou-se necessário investigar quais íons faziam parte desta camada, visto que, às vezes, não ocorria nucleação ou não se conseguia obter o crescimento desejado da camada de HA, além de alguns íons serem substituídos por outros.

O presente trabalho investigou a modificação da superfície da liga Ti-6Al-4V com aplicação de feixe de laser Nd:YAG. Parâmetros do laser, como o tempo de exposição, potência, velocidade de varredura, comprimento de onda, frequência, número de passo e área de exposição, foram mantidos fixos, variando-se o espaço entre varreduras (espaçamento da matriz) com o objetivo de verificar essa influência na topografia da liga. Em uma segunda etapa, foi avaliado o efeito dessa modificação superficial sobre a deposição de apatitas pelo método biomimético, visando a sua aplicação clínica.

\section{MATERIAIS E MÉTODOS}

\subsection{Liga Ti-6Al-4V}

A composição química nominal da liga utilizada e a estabelecida pela Norma ASTM F136 estão relacionadas na Tabela 1. O metal utilizado para estudo, Ti-6Al-4V, foi cortado em pequenas barras com dimensões de 10 mm x 7,5 mm, com o auxílio de uma máquina ISOMET 1000 do Instituto de Química - Unesp - Araraquara SP, lixadas com uma lixa de 80 mesh.

Tabela 1: Composição química nominal da liga Ti-6Al-4V e a estabelecida pela ASTM F136 [13].

\begin{tabular}{l|r|r|r|r|r|r|r|r|r}
\hline ELEMENTOS & $\mathbf{N}$ & $\mathbf{C}$ & $\mathbf{H}$ & $\mathbf{F e}$ & $\mathbf{0}$ & $\mathbf{A l}$ & $\mathbf{V}$ & $\mathbf{S}$ & $\mathbf{T i}$ \\
\hline ASTM F136 & 0,006 & 0,020 & 0,004 & 0,18 & 0,19 & 6,1 & 4,0 & - & balanço \\
\hline LIGA TI-6AL-4V & 0,005 & 0,08 & 0,00125 & 0,025 & 0,13 & 6,0 & 4,0 & - & balanço \\
\hline
\end{tabular}

\subsection{Irradiação da superfície do titânio com laser}

As amostras de Ti-6Al-4V, antes de submetidas ao tratamento com laser, foram lixadas com lixa de 2000 mesh para homogeneizar sua superfície. As amostras foram irradiadas com o laser Nd:YAG. A Tabela 2 mostra os parâmetros dos feixes de laser utilizados. Após a aplicação do feixe de laser, os substratos foram limpos no ultrassom com soluções de detergente neutro, água Milli-Q e acetona-etanol (1:1), e posteriormente secos em estufa à temperatura de $50^{\circ} \mathrm{C}$.

Tabela 2: Parâmetros dos feixes de laser

\begin{tabular}{l|r|r}
\hline PARÂMETROS & AMOSTRA A & AMOSTRA B \\
\hline POTÊNCIA (W) & 100 & 100 \\
\hline VELOCIDADE DE VARREDURA (mm/s) & 300 & 300 \\
\hline PASSO & 1 & 1 \\
\hline ESPAÇAMENTO DA MATRIZ (cm) & $\mathbf{0 , 0 1}$ & $\mathbf{0 , 0 2}$ \\
\hline FREQUÊNCIA (KHz) & 35 & 35 \\
\hline
\end{tabular}

\subsection{Recobrimento de apatita sobre a liga Ti-6Al-4V}

Após a limpeza, as amostras foram imersas em uma solução de $\mathrm{NaOH} 5 \mathrm{~mol} . \mathrm{L}^{-1}$ por um período de $24 \mathrm{~h}$, com o objetivo de se formar uma camada de hidrogel de titanato de sódio, que induz mais satisfatoriamente a formação de HA [15]. Após o tratamento em solução de $\mathrm{NaOH}$, os substratos foram imersos em uma solução de SBF (com concentração similar ao plasma sanguíneo) para uma nucleação mais lenta e organizada da apatita a ser depositada. Em seguida, os substratos foram imersos durante 4 dias a $37{ }^{\circ} \mathrm{C}$ em uma solução de 1,5 SBF. Após o processo de recobrimento, essas soluções foram submetidas ao tratamento térmico por um período de $1 \mathrm{~h}$ a $600{ }^{\circ} \mathrm{C}$, para melhor densificação da camada e aderência. A Tabela 3 apresenta as concentrações iônicas do plasma sanguíneo e de todas as soluções utilizadas neste processo. 
Tabela 3: Concentrações iônicas das soluções utilizadas para o recobrimento de apatitas (mmol.L $\left.\mathrm{L}^{-1}\right)$

\begin{tabular}{l|r|r|r|r|r|r|r|r}
\hline & $\mathbf{N a}$ & $\mathbf{K}$ & $\mathbf{C a}^{2+}$ & $\mathbf{M g}^{\mathbf{}^{+}}$ & $\mathbf{H C O}_{3}{ }^{2-}$ & $\mathbf{C L}^{-}$ & $\mathbf{H P O}_{4}{ }^{2-}$ & $\mathbf{S O}_{4}{ }^{{ }^{-}}$ \\
\hline PLASMA SANGUÍNEO & 142,0 & 5,0 & 2,5 & 1,5 & 27,0 & 103,0 & 1,0 & 0,5 \\
\hline SBF & 142,0 & 5,0 & 2,5 & 1,5 & 4,2 & 148,0 & 1,0 & 0,5 \\
\hline $\mathbf{1 , 5}$ SBF & 213,0 & 7,5 & 3,8 & 2,3 & 6,3 & 223,0 & 1,5 & 0,75 \\
\hline
\end{tabular}

\subsection{Caracterização das Amostras}

Todas as amostras com e sem recobrimento foram caracterizadas por Microscopia Eletrônica de Varredura, em um microscópio LEO modelo 440, acoplado a um analisador por dispersão de energia de Si (Li) com janela de Be, modelo 760 e resolução de 133 eV- EDS, do Instituto de Química - USP/São Carlos. As amostras também foram analisadas em um difratômetro de raiosX Siemens D5000, varredura angular de $10^{\circ}$ a $50^{\circ}$, com passo de 0,02 (20). O tempo de passo foi de 10 segundos para cada amostra, na montagem de Bragg-Brentano, utilizando-se radiação de $\mathrm{Cu}(\mathrm{k} \alpha 1)$, do Instituto de Química da Unesp de Araraquara-SP; e por espectroscopia no infravermelho - IV, espectrômetro Perkin Elmer, FT-IR Spectrometer-Spectrum 2000, de reflectância difusa Drift Collector. As fases presentes foram comparadas com as fichas padrões do JCPDS [16].

\section{RESULTADOS E DISCUSSÃO}

A Figura 1 apresenta as micrografias das amostras A e B irradiadas e recobertas pelo método biomimético. Verificou-se grande diferença na morfologia das superfícies, proporcionada pelas diferentes características dos tratamentos. As Figuras 1(a) e 1(c) mostram as superfícies das amostras A e B, respectivamente, nas quais pode-se observar a formação de uma morfologia com diferentes rugosidades. As Figuras 1(b) e 1(d) mostram as micrografias das amostras A e B, respectivamente, submetidas ao tratamento biomimético. Verificou-se que, em ambas as amostras, o recobrimento procedeu-se de forma satisfatória. A superfície submetida com espaçamento de matriz de $0,01 \mathrm{~cm}$ proporcionou a formação de microesferas de hidroxiapatita com distribuição de tamanho entre $5 \mu \mathrm{m}$ e $15 \mu \mathrm{m}$, conforme mostrado na Figura 1(c). Entretanto, para a amostra B, Figura 1(d), verificou-se a formação de um recobrimento não homogêneo sem a presença de microesferas.
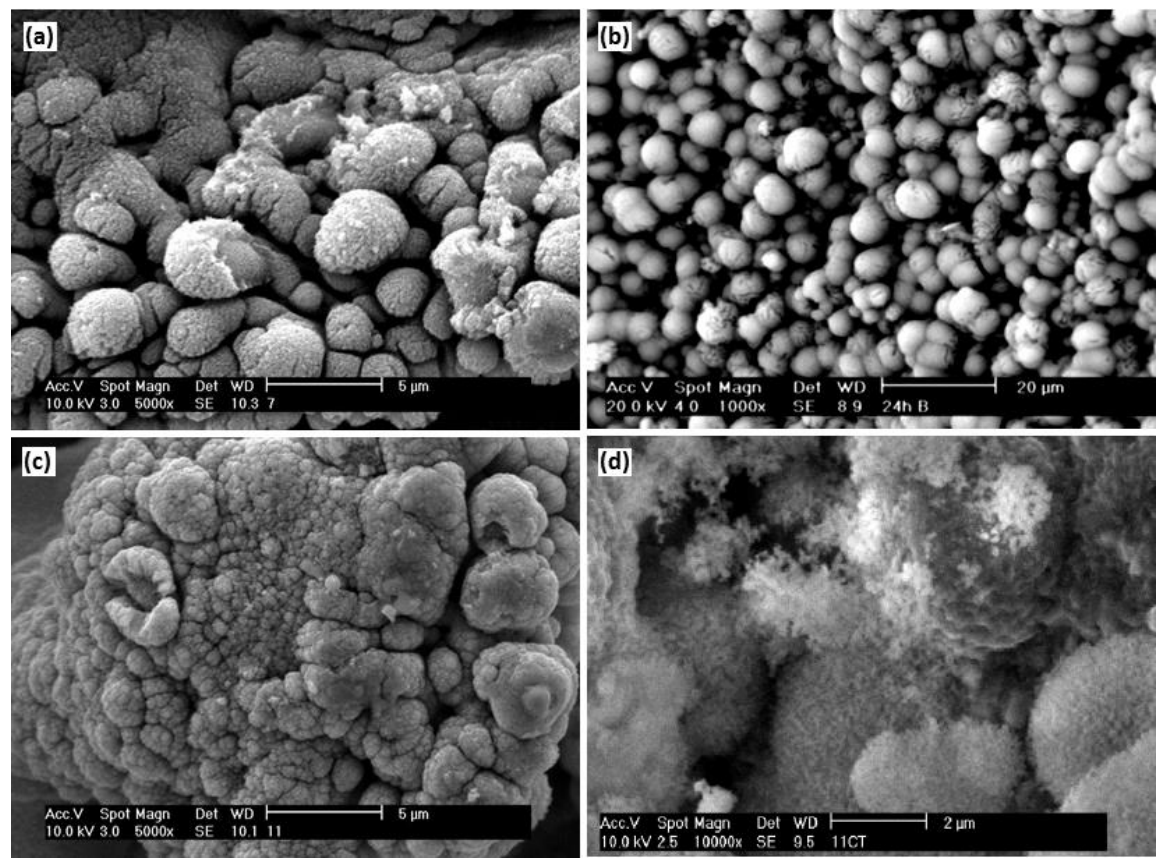

Figura 1: Microscopias eletrônicas de varredura da superfície das amostras da liga de titânio modificadas pelos tratamentos com laser Nd:YAG e método biomimético. (a) Ti-6AL-4V irradiada pelo laser com espaçamento da matriz de $0,01 \mathrm{~cm}$ (amostra A); (b) amostra A submetida à solução de SBF e tratamento térmico a $600{ }^{\circ} \mathrm{C}$; (c) Ti-6AL-4V 
irradiada pelo laser com espaçamento da matriz de 0,02 cm (amostra B); (d) amostra B submetida à solução de SBF e tratamento térmico a $600{ }^{\circ} \mathrm{C}$.

Analisando-se o espectro de EDS das amostras A e B, Figura 2(a) e 2(c), respectivamente, verificouse a presença de íons cálcio e fósforo com picos relativamente intensos, além um pico de titânio e de magnésio. O pico de magnésio é provavelmente devido ao fato de a solução utilizada para o recobrimento conter esses íons que foram incorporados na estrutura das apatitas. O pico de titânio é devido à presença de tal elemento no substrato utilizado. Já a não aparição de picos de alumínio e vanádio é consequência de um recobrimento bastante espesso e do fato de esses materiais estarem em pequenas quantidades no material de trabalho. A relação cálcio/fósforo obtida foi de 1,51 para a amostra A e 1,45 para a amostra B, enquanto, para a hidroxiapatita pura, a relação é de 1,67. As Figuras 2(b) e 2(d) apresentam os difratogramas obtidos das amostras A e B tratadas termicamente à temperatura de $600{ }^{\circ} \mathrm{C}$. Pode-se observar, em ambos difratogramas, a formação de uma mistura de fase contendo hidroxiapatida e fosfato de tricálcio.

A Figura 3 mostra os espectros no infravermelho das amostras A e B em comparação com o pó de HA comercial. A Tabela 4 apresenta as frequências de absorção no infravermelho para os compostos à base de fosfato de cálcio. De acordo com STOCH et al., a hidroxiapatita com fórmula molecular $\mathrm{Ca}_{10}\left(\mathrm{PO}_{4}\right)_{6}(\mathrm{OH})_{2}$ possui absorções de modo de vibrações para os grupos fosfato e hidroxilas. Em apatitas biológicas, alguns íons $\mathrm{PO}_{4}{ }^{3-}$ são substituídos por íons $\mathrm{CO}_{3}{ }^{2-}$ e o infravermelho é muito sensível a essas pequenas substituições. Portanto, mesmo uma quantidade muito pequena de carbonato substituído pode ser detectada [17]. Analisando o espectro da amostra A, observou-se um estiramento em $475 \mathrm{~cm}^{-1}$ e $1197 \mathrm{~cm}^{-1}$, referentes ao grupo $\mathrm{PO}_{4}$; a banda em $630 \mathrm{~cm}^{-1}$ é atribuída à ligação $\mathrm{OH}$ da $\mathrm{HA}$; e os estiramentos em $848 \mathrm{~cm}^{-1}$ e $987 \mathrm{~cm}^{-1}$ são referentes ao grupo $\mathrm{P}-\mathrm{OH}$. Na amostra $\mathrm{B}$, observaram-se também estiramentos do grupo $\mathrm{PO}_{4} \mathrm{em} 475 \mathrm{~cm}^{-1}$ e $1200 \mathrm{~cm}^{-1}$; a banda em $630 \mathrm{~cm}^{-1}$ é atribuída ao modo de vibração do grupo hidroxila; e o estiramento em $997 \mathrm{~cm}^{-1}$ é atribuído ao grupo P-OH.
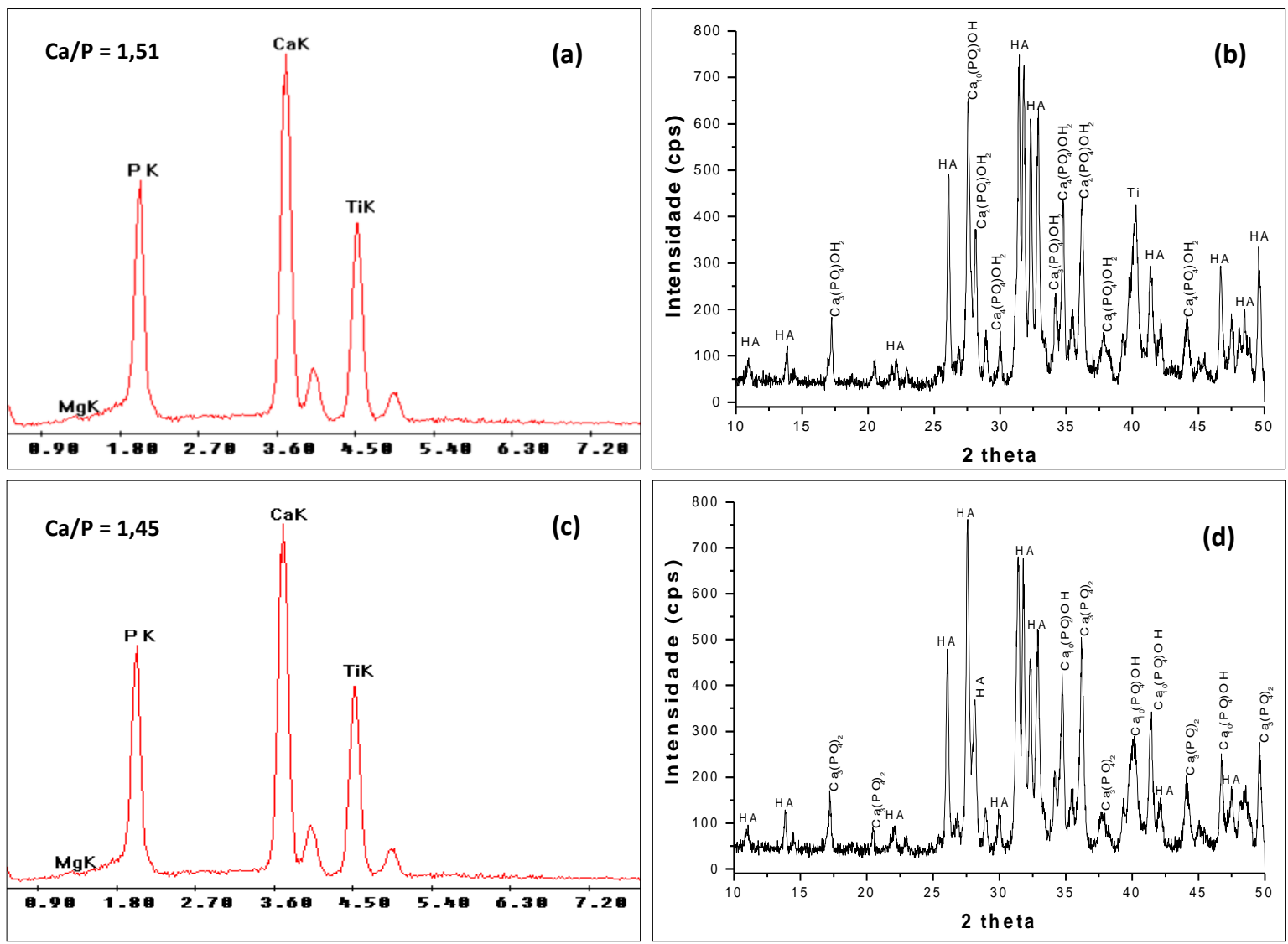

Figura 2: Os quadros (a) e (c) são espectros de EDS das amostras A e B, respectivamente; (b) e (d) correspondem aos difratogramas das amostras A e B pelo método biomimético, respectivamente. 


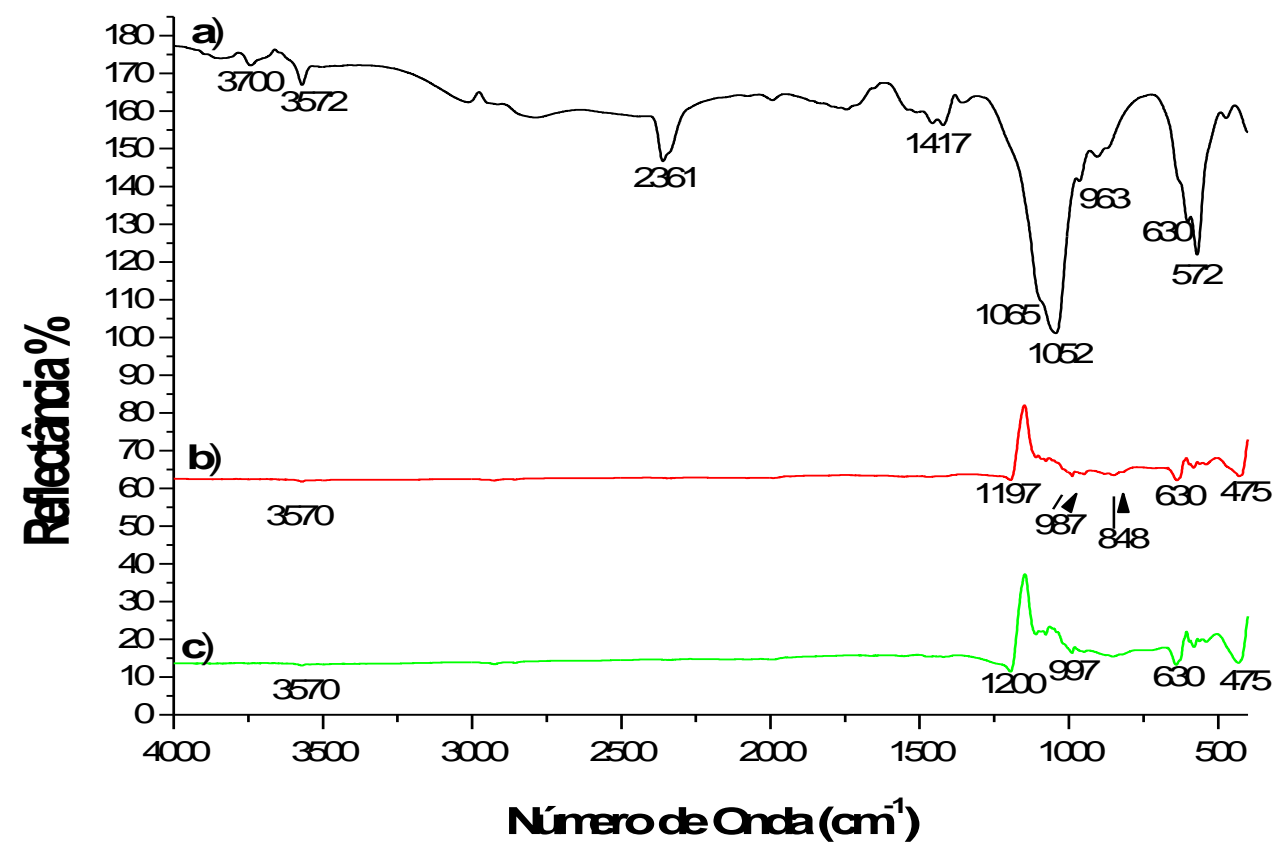

Figura 3: Espectros no infravermelho da HA comercial (a) e das amostras A (b) e B (c).

Tabela 4: Frequências de absorção no infravermelho características dos fosfatos de cálcio [18]

\begin{tabular}{l|l}
\hline MODOS DE VIBRAÇÃO & NÚMERO DE ONDA $\left(\mathbf{C m}^{-1}\right)$ \\
\hline $\mathrm{OH}^{-}$(livre) & 3572,630 \\
\hline $\mathrm{PO}_{4}{ }^{3-}$ & $474,562,580,640$ e $960-1200$ \\
\hline $\mathrm{P}-\mathrm{OH}$ & 527,870 e $910-1040$ \\
\hline $\mathrm{CO}_{3}{ }^{2-}$ (substituição do tipo A) & 880,1450 e 1514 \\
\hline $\mathrm{CO}_{3}{ }^{2-}$ (substituição do tipo B) & 870,1412 e 1465 \\
\hline
\end{tabular}

\section{CONCLUSÃO}

As modificações de superfície da liga Ti-6Al-4V pelo laser Nd:YAG mostraram-se propícias para a nucleação de apatitas, uma vez que os resultados do recobrimento via método biomimético mostraram-se satisfatórios, tendo as duas amostras estudadas propiciado a formação de uma mistura de fases hidroxiapatita e fosfato tricálcico. Verificou-se que os melhores resultados foram obtidos para a amostra A, indicando que o espaçamento da matriz influencia na morfologia do recobrimento. Assim, pode-se concluir que o espaçamento da matriz de $0,01 \mathrm{~cm}$ propicia condições mais favoráveis à formação e ancoragem de microesferas de hidroxiapatita. Portanto, como a irradiação a laser caracteriza-se por ser um processo limpo e reprodutível, o uso da ferramenta a laser na produção de superfícies ativas para a deposição de biocerâmicas mostrou-se viável, bem como o substrato após o recobrimento final possui potencial para aplicações médicas.

\section{AGRADECIMENTOS}

Os autores agradecem ao CNPq e à FAPESP pelo suporte financeiro.

\section{BIBLIOGRAFIA}

[1] INTERNATIONAL ORGANIZATION FOR STANDARD, ISO 10993: biological evaluation of medical devices, http://www.iso.org/iso/en/ISOOnline.frontpage, Acessado em maio 2011. 
[2] BRANEMARK, P.I., ZARB, G.A., ALBREKTSSON, T., "Prótesis tejido - integradas: la oseointegración en la odontología clínica", Berlin: Quintessenz Verlags-Gmbh, Berlin, 1987.

[3] THOMAS, K.A., JAY, J.F., COOK, S.D., "The effect on surface macrotexture and hydroxyapatite coating on the mechanical strengths and histologic profiles of titanium implant materials", Journal of Biomedical Materials Research, v. 21, pp. 1395-1441, 1987.

[4] GUASTALDI, A.C., "Engenharia de superfície do titânio para aplicações biomédicas", Metalurgia \& Materiais, v. 59, pp. 442, 2003.

[5] STORZ, O., GAUSTHUBER, H., WOYDT, M., "Tribological properties of thermal-sprayed Magnélitype coatings with different stoichiometries $\left(\mathrm{Ti}_{\mathrm{n}} \mathrm{O}_{2 \mathrm{n}-1}\right)$ ", Surface and Coating Technology, v. 140, n. 2, pp. 76-81, 2001.

[6] GROSS, K.A., BERNDT, C.C., GOLDSCHLAG, D.D., et al., "In vitro changes of hydroxyapatite coatings", International Journal of Oral \& Maxillofacial Implants, v. 12, n. 5, pp. 589-597, 1997.

[7] KAWACHI, E.Y., BERTRAN, C.A., REIS, R.R., et al., "Biocerâmicas: tendências e perspectivas de uma área interdisciplicar", Química Nova, v. 23, n. 4, pp. 518-522, 2000.

[8] NAKAZAWA, T., "Inorganic Phosphate materials", Elsevier, Tokyo, 1989.

[9] RIGO, E.C.S., OLIVEIRA, L.C., SANTOS, L.A., et al., "Biomimetric method for obtaining of hydroxyapatite", Advanced Powder Technology Materials Science Forum, n. 299, pp. 99-103, 1999.

[10] ABE, Y., KOKUBO, T., YAMAMURO, T., "Apatite coatings on ceramics, metals and polymers utilising a biological process", Journal of Materials Science: Materials in Medicine, v. 1, n. 4, pp. 233-238, 1990.

[11] BARRERE, F., LAYROLLE, P., VAN BLITTERSWIJK, C.A., et al., "Biomimetic calcium phosphate coatings on Ti-6Al-4V: a crystal growth study of octacalcium phosphate and inhibition by $\mathrm{Mg}^{2+}$ and $\mathrm{HCO}^{3-»,}$, Bone, v. 25, n. 2, pp. 107S-111S, 1999.

[12] SHI, J., DING, C., WU, Y., "Biomimetic apatite layers on plasma-sprayed titanium coatings after surface modification", Surface \& Coating Technology, v. 137, n. 1, pp. 97-103, 2001.

[13] ASTM Handbook, Materials Characterization, Ninth Edition, 1992.

[14] WETTER, N.U., ROSSI, W., ICS Lectures on industrial applications of lasers, Industrial applications of lasers, 1 ed., Vienna, Unido Publication, 2000.

[15] KOKUBO, T., "Apatite formation on surfaces of ceramics, metals and polymers in body environment", Acta Materialia, v. 46, n. 7, pp. 2519, 1998.

[16] JOINT COMMITTEE POWDER DIFFRACTION STANDARD, Diffraction data base, Newton Square: International for Diffraction Data, 2003, 1 CD-ROM.

[17] STOCH, A., JASTRZEBSKI, W., BROZEK, A., et al., "FTIR absorption-reflection of biomimetic growth of phosphates on titanium implants", Journal of Molecular Structure, v. 555, n. 1/3, pp. 375-382, 2000.

[18] SILVERSTEIN, R.M., BASSLER, G.C., MORRIL, T.C., Identificação espectrométrica de compostos orgânico, 5 ed. Rio de Janeiro, Editora Guanabara Koogan, 1994. 460 p. 